\title{
Unsedated Transnasal Endoscopy: A Safe, Well-Tolerated and Accurate Alternative to Standard Diagnostic Peroral Endoscopy
}

\author{
Rebecca K. Grant ${ }^{1} \cdot$ William M. Brindle ${ }^{1} \cdot$ Alexander R. Robertson $^{2} \cdot$ Rahul Kalla $^{1} \cdot$ John N. Plevris ${ }^{1}$
}

Received: 15 October 2021 / Accepted: 30 January 2022 / Published online: 3 March 2022

(c) The Author(s), under exclusive licence to Springer Science+Business Media, LLC, part of Springer Nature 2022

\begin{abstract}
Diagnostic unsedated transnasal endoscopy (UTNE) has been proven to be a safe and well-tolerated procedure. Although its utilization in the United Kingdom (UK) is increasing, it is currently available in only a few centers. Through consideration of recent studies, we aimed to perform an updated review of the technological advances in uTNE, consider their impact on diagnostic accuracy, and to determine the role of uTNE in the COVID-19 era. Current literature has shown that the diagnostic accuracy of uTNE for identification of esophageal pathology is equivalent to conventional esophagogastroduodenoscopy (cEGD). Concerns regarding suction and biopsy size have been addressed by the introduction of TNE scopes with working channels of $2.4 \mathrm{~mm}$. Advances in imaging have improved detection of early gastric cancers. The procedure is associated with less cardiac stress and reduced aerosol production; when combined with no need for sedation and improved rates of patient turnover, uTNE is an efficient and safe alternative to cEGD in the COVID-19 era. We conclude that advances in technology have improved the diagnostic accuracy of uTNE to the point where it could be considered the first line diagnostic endoscopic investigation in the majority of patients. It could also play a central role in the recovery of diagnostic endoscopic services during the COVID-19 pandemic.
\end{abstract}

Keywords Trans-nasal endoscopy · Barrett's esophagus · Gastric cancer · Esophageal varices · Eosinophilic esophagitis

\section{Introduction}

Use of unsedated transnasal endoscopy (uTNE) has grown since its first description in 1994 [1], with the number of procedures performed in Japan in particular gaining traction in recent years [2]. However, conventional (peroral) esophagogastroduodenoscopy (cEGD) remains the predominant

Rebecca K. Grant

rebecca.x.grant@nhslothian.scot.nhs.uk

William M. Brindle

william.m.brindle@nhslothian.scot.nhs.uk

Alexander R. Robertson

alexander.robertson7@nhs.net

Rahul Kalla

rahul.kalla@nhslothian.scot.nhs.uk

John N. Plevris

j.plevris@ed.ac.uk

1 The Centre for Liver and Digestive Disorders, Royal Infirmary of Edinburgh, Edinburgh, UK

2 The Edinburgh IBD Unit, Western General Hospital, Edinburgh, UK diagnostic tool in most centers worldwide, including the United Kingdom (UK), as demonstrated in recently published data obtained from the National Endoscopy Database [3].

uTNE is performed using ultrathin endoscopes $(6 \mathrm{~mm}$ diameter or less) via the nasal route. This has the major advantage of bypassing the regions of maximum sensitivity within the oral cavity, which can trigger the gag reflex (mediated by cranial nerves IX (glossopharyngeal) and X (vagus)); these areas are the fauces, base of the tongue, palate, uvula and posterior pharyngeal wall. (Fig. 1) Reduction in gagging results in better procedure tolerance and patient satisfaction $[4,5]$.

There have been two comprehensive reviews on the use of uTNE compared to cEGD, both completed approximately six years ago. The systematic review by Sami et al. [6] (2016) showed that, while the technical success rate of uTNE (based on a meta-analysis of 3565 patients) was equivalent to that of cEGD, it also had higher patient acceptance and tolerability, and provoked significantly less cardiovascular stress than cEGD. Although the reliability of UTNE in the detection of early gastric cancers 
Fig. 1 Peroral versus transnasal routes of insertion. a Peroral route with trigger points for gag reflex. Highlighted (a) palate; (b) uvula; (c) fauces; (d) base of tongue; (e) posterior pharyngeal wall. b Transnasal route. Illustrations courtesy of Mr Stephen Liddell, Creative 82
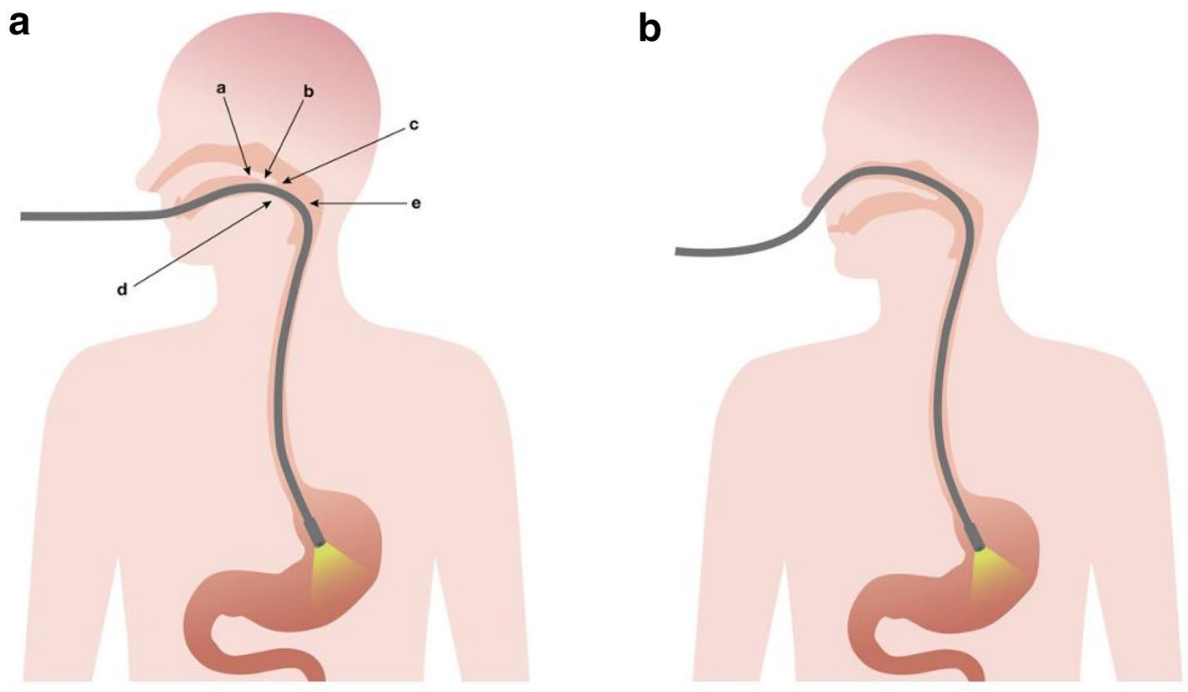

was not investigated in the meta-analysis, the authors did acknowledge that concerns regarding diagnostic accuracy had been reported [6].

The narrative review of Parker et al. [7] (2016) summarized the many advantages of uTNE, including improved patient tolerance, with no strong evidence for inferiority in the detection/assessment of Barrett's esophagus, established gastric cancer or gastro-esophageal reflux disease (GERD). The potential cost-savings were also highlighted. They also identified the concern in relation to the diagnosis of early gastric cancer.

With the advancement of endoscopic technologies and the emergence of new data, in addition to the advent of the COVID-19 pandemic, there is a pressing need to reconsider the indications for diagnostic upper gastrointestinal (GI) endoscopy, as well as the route (oral versus nasal) by which the procedure is performed. In particular, the COVID-19 pandemic has not only created the need to reduce aerosol generation in endoscopic procedures, but has also put endoscopic services under immense pressure worldwide.

In this review, we aim to provide a comprehensive update of the literature regarding the utility of diagnostic uTNE, to summarize the technological advances in endoscope design in this period, and to discuss the reasons why we are yet to move to uTNE as our test of choice for diagnostic upper GI endoscopy.

\section{Performing Transnasal Endoscopy- Practicalities and Limitations}

uTNE is typically performed with the patient in the left lateral position (as in cEGD), although it is also possible for patients to sit upright in a chair. Adequate pretreatment techniques are paramount in ensuring optimum comfort during the procedure; the approach however is currently not standardized and may vary between centers [8]. It has been observed that most discomfort from uTNE is related to the insertion of the scope into the nostril and when the endoscope is in contact with the posterior pharyngeal wall, therefore a combination of topical lidocaine gel to the nostrils and lidocaine spray to the oropharynx has been reported as being best tolerated [9] and has the added benefit of easy conversion to the peroral approach if needed [8]. The use of a decongestant such as xylometazoline or phenylephrine with this combination is also helpful in aiding nasal insertion due to its effect on turbinate shrinkage [7]. In our unit, we use a combination of lidocaine $5 \% /$ phenylephrine $0.5 \%$ nasal spray.

Once adequate pretreatment has been performed, the well-lubricated TNE scope is passed through the nasal cavity (either below or above the inferior turbinate) to the posterior pharynx. (Fig. 2) Upon passing the upper esophageal sphincter, the procedure continues as in cEGD. On traversing the nasal cavity, it is important to advance slowly and to avoid contact with the nasal septum due to its high sensitivity [7] and risk of contact bleeding.

Epistaxis is reported as the most frequent complication of TNE, occurring in approximately $1-5 \%$ of cases in clinical studies, but it is predominantly mild and self-limiting [8]. A 2017 Japanese study identified patients who were younger and female at increased risk of epistaxis [10]. Official guidelines are not in existence, however, uTNE should be avoided in patients with altered nasal anatomy, severe coagulopathy, and previous nasal trauma or nasal surgery in order to limit epistaxis risk. Other rare complications include mucous discharge, dizziness, headache, earache, eye discharge and sinusitis; however these have been small in number $[8,11]$ and are therefore of limited significance. (Table 1) In $2-6 \%$ of cases there is failure 


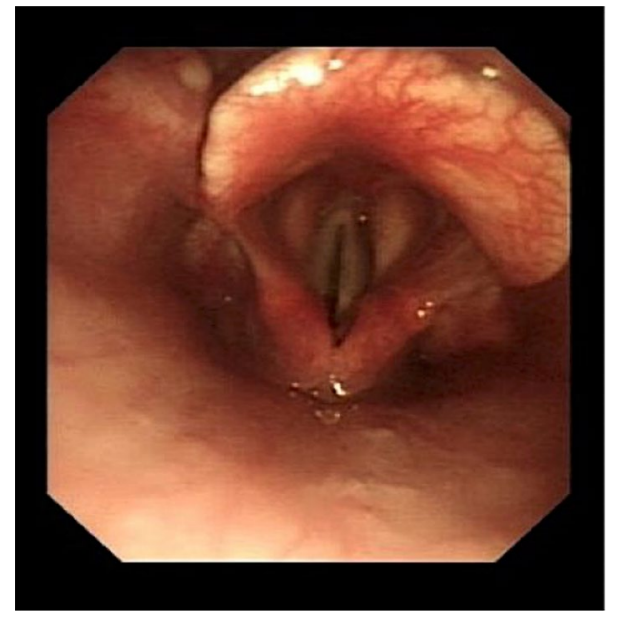

Fig. 2 Visualization of the epiglottis

of nasal intubation due to narrow nasal passages [12]; in the past we routinely assessed nasal patency pretreatment with a 14/16 French nasal catheter prior to attempting intubation, this, however, is no longer considered necessary, as intubation can be achieved effectively under direct visualization.

\section{Technological Advances}

Current commercially available TNE endoscopes now have a distal end diameter of between 4.9 and $5.9 \mathrm{~mm}$, allowing for a working channel of between 2.0 and $2.4 \mathrm{~mm}$ (with the wider channels available on the slightly wider endoscopes). (Table 2) This contrasts with standard cEGD endoscopes that have an insertion tube outer diameter that varies from 9.4 to $9.9 \mathrm{~mm}$ and a $2.8 \mathrm{~mm}$ working channel [7]. (Fig. 3) The narrower working channel has led to concern over the yield of biopsies which can be retrieved. However, although the biopsies taken are smaller, with less mucosal depth, it has been demonstrated that their diagnostic yield is comparable to those through a cEGD endoscope [13].

Narrower channel width has traditionally resulted in reduced suction, less effective washing and reduced availability of accessories [7]. However, the development of TNE endoscopes with a biopsy channel of $2.4 \mathrm{~mm}$ (compared with $2 \mathrm{~mm}$ previously) has led to improvements in all of

Table 1 Advantages and limitations of diagnostic TNE

\begin{tabular}{ll}
\hline Advantages & Limitations \\
\hline High patient satisfaction, well-tolerated with reduced gagging & $\begin{array}{c}\text { Not suitable for patients with altered nasal anatomy, history of pre- } \\
\text { vious nasal trauma/nasal surgery or severe coagulopathy } \\
\text { Risk of epistaxis }\end{array}$ \\
$\begin{array}{l}\text { Reduced cardiovascular stress compared to cEGD } \\
\text { No sedation and no need for routine oxygen administration removes risk of } \\
\text { associated side effects, allows for immediate recovery, quicker through- } \\
\text { put of patients and less personnel necessary }\end{array}$ & $\begin{array}{l}\text { Risk of nasal pain and discomfort } \\
\begin{array}{l}\text { Pedential to be used in community outpatient setting } \\
\text { mask }\end{array}\end{array}$ \\
$\begin{array}{l}\text { Diagnostics not inferior compared to cEGD } \\
\text { Potential for failure of nasal intubation due to narrow nasal passages }\end{array}$ \\
\hline
\end{tabular}

Table 2 Technical specifications of current uTNE endoscopes (2021)

\begin{tabular}{|c|c|c|c|c|c|c|c|}
\hline Manufacturer & Camera CCD & $\begin{array}{l}\text { Diameter DED/ } \\
\text { ITOD }(\mathrm{mm})\end{array}$ & $\begin{array}{l}\text { Optical } \\
\text { range }(\mathrm{mm})\end{array}$ & $\begin{array}{l}\text { Field of } \\
\text { view }\left({ }^{\circ}\right)\end{array}$ & $\begin{array}{l}\text { Angulation: U / D } \\
/(\mathrm{L} \& \mathrm{R})\left(^{\circ}\right)\end{array}$ & $\begin{array}{l}\text { Working chan- } \\
\text { nel }(\mathrm{mm})\end{array}$ & Image enhancement \\
\hline \multicolumn{8}{|l|}{ FUJIFILM } \\
\hline EG-580NW2 & Color & $5.8 / 5.9$ & $3-100$ & 140 & $210 / 90 / 100$ & 2.4 & BLI/LCI \\
\hline EG-740N & & $5.8 / 5.9$ & $3-100$ & 140 & $219 / 90 / 100$ & 2.4 & BLI/LCI \\
\hline \multicolumn{8}{|l|}{ OLYMPUS } \\
\hline GIF-XP290N & Monochrome & $5.4 / 5.8$ & $3-100$ & 140 & $210 / 90 / 100$ & 2.2 & NBI \\
\hline GIF-H190N & & $5.4 / 5.8$ & $3-100$ & 140 & $210 / 90 / 100$ & 2.2 & NBI \\
\hline \multicolumn{8}{|l|}{ PENTAX } \\
\hline EG16-K10 & Color & $5.2 / 5.4$ & $4-100$ & 140 & $210 / 120 / 120$ & 2.0 & iSCAN \\
\hline
\end{tabular}

$C C D$ charge-coupled device, $D E D$ distal end diameter, ITOD insertion tube outer diameter, $U$ up, $D$ down, $L$ Left, $R$ right, $B L I$ blue light imaging, $L C I$ linked color imaging, $N B I$ narrow band imaging 


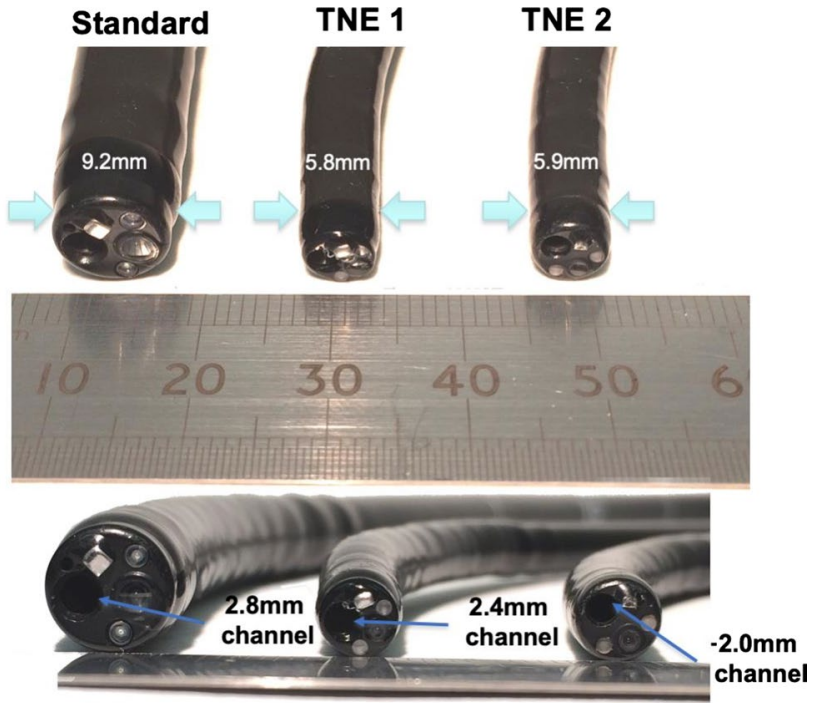

Fig. 3 Comparison of tip and biopsy channel diameter of a standard endoscope versus transnasal endoscopes. Illustration courtesy of Dr S Inglis, Department of Medical Physics, The Royal Infirmary of Edinburgh

these areas. Fluid and air suction flow has doubled, and more accessories can be used, including larger biopsy forceps.

Previously, there have been concerns regarding diagnostic performance of UTNE due to poorer views and reduced flexibility when compared to cEGD [7]. Optics have, however, continued to improve with more modern technologies, and TNE endoscopes from Olympus (Olympus Corporation, Tokyo, Japan), Fujifilm (Fujifilm Corporation, Tokyo, Japan) and Pentax (Pentax Corporation, Tokyo, Japan) now offer a $140^{\circ}$ field of view as in cEGD endoscopes (Fig. 4). Image enhanced endoscopy or virtual chromoendoscopy have been shown to enhance gastric cancer detection, sensitivity, specificity and accuracy in depressed lesions when compared with white light $[14,15]$. TNE endoscopes are available with image enhancement from several manufacturers, including blue light imaging (BLI) and linked color imaging (LCI) from Fujifilm, narrow band imaging (NBI) from Olympus, and iSCAN from Pentax. These image enhancement modalities are a step toward computer-aided diagnostics (CADx) and artificial intelligence (AI), both of which are emerging into real-world practice [15].

An oral approach is still favored when therapy or hemostasis is likely to be required, although, as technology continues to advance and further evidence emerges [7], there is an increasing role for therapeutic TNE (such as for strictures [16], percutaneous endoscopic gastrostomy [17-19] and feeding tube placement [20], endoscopic submucosal dissection [21, 22] and Botulinum toxin (Botox) injections [23]). Recent studies have also explored the use of uTNE in the pre-screening of bariatric surgery patients, with results suggesting that it is a well-tolerated examination and is an appropriate choice in morbidly obese patients due to minimal cardiovascular stress being induced [24, 25]. A 2021 study by Abe et al. [26] also considered the safety and efficiency of uTNE in gastrointestinal bleeding; results indicated that it was an efficient alternative to cEGD in achieving initial hemostasis in cases with severe strictures on the oral side of the bleeding point. The authors do acknowledge that the development of hemostatic devices that can be used specifically with TNE endoscopes is required.

\section{Diagnostics (Table 3)}

\section{Esophageal}

\section{Barrett's Esophagus}

Endoscopy is a key diagnostic tool for Barrett's esophagus. While screening of patients with gastro-esophageal reflux symptoms for Barrett's esophagus is not currently recommended by the British Society of Gastroenterology (BSG) [27], it is acknowledged that uTNE may have a role to play if we were to introduce a screening program among a highrisk population (e.g., male, $>50$, white race, obese). When the BSG guidelines were written in 2013 it was felt that
Fig. 4 Advances in TNE field of view. $120^{\circ}$ (left image) versus $140^{\circ}$ field of view (right image) of esophagogastric junction captured by two different TNE endoscopes using the same Endoscopy Video Processor
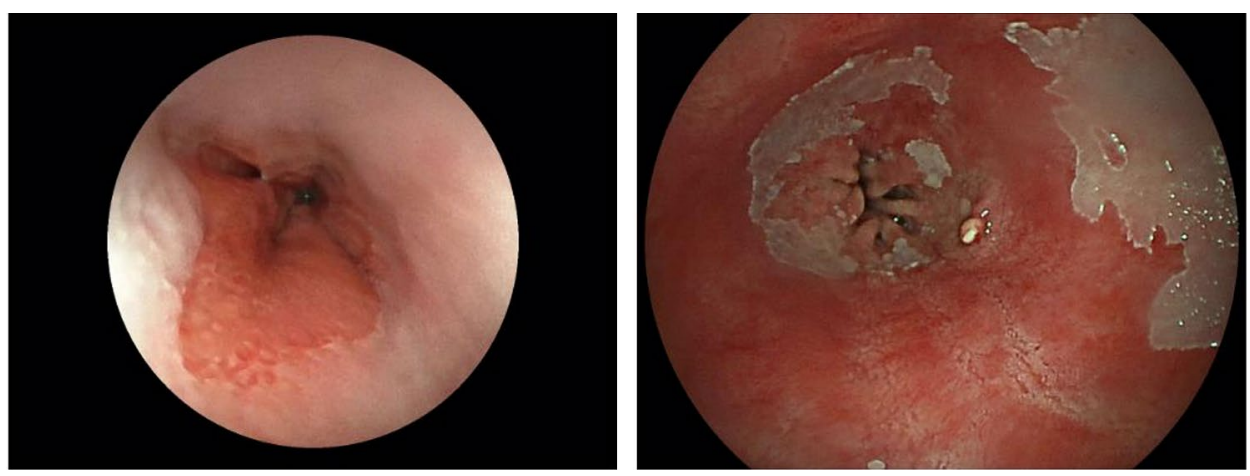
more evidence was required before screening using uTNE could be recommended; studies involved small numbers of patients, often did not have a diagnosis of Barrett's as the primary end point, and there were also concerns regarding biopsy size.

Subsequently, a prospective randomized control trial by Sami et al. in 2015 [28] demonstrated comparable clinical effectiveness of uTNE to cEGD in the endoscopic diagnosis of Barrett's esophagus. This study comprised 209 patients and reported good safety and participation rates among the cohort. The acquisition of biopsies was, however, noted as a limitation, with a mobile van uTNE rate of $79.0 \%$ and clinic uTNE rate of $83.3 \%$ compared to a cEGD rate of $100.0 \%$. This was attributed to the inability of the biopsy sheath to be advanced through the biopsy channel of the TNE endoscope due to narrow nasal passages; this issue will most likely now have been resolved with the latest TNE endoscopes with a biopsy channel of $2.4 \mathrm{~mm}$. Positive results regarding the use of uTNE for screening were also reported by Shariff et al. in 2016 [29]; in this study disposable endoscopes were used, suggesting the potential for use in a community setting.

The well-documented [7] reduction in stimulation of the gag reflex during uTNE compared to cEGD makes it easier to define anatomical structures, determine the length of the Barrett's segment, as well as perform targeted biopsies with higher accuracy. (Fig. 1) This, in addition to the larger biopsy channel size of current technology, and increased tolerance allowing for 4-quadrant biopsy sampling, suggest that UTNE is a suitable tool for Barrett's surveillance. A 2015 Japanese study [30] concluded that uTNE (with white light imaging (WLI) and NBI) could effectively be used in the surveillance of short segment Barrett's. The development of LCI has been shown to improve visibility in the diagnosis of short segment lesions [31]; however there are currently no data on its general use in Barrett's surveillance (in either cEGD or uTNE). Furthermore, the emerging use of cytosponge [32], if combined with uTNE for patients needing further endoscopic assessment and biopsies, may potentially improve the screening and surveillance of Barrett's and reduce the need for sedated cEGD; data are, however, required before implementing such an investigation pathway.

\section{Esophageal Varices}

cEGD is recognized as the gold standard in the diagnosis of esophageal varices and, prior to the COVID-19 pandemic, the BSG advised that it be performed every 2-3 years in all patients with cirrhosis and annually in those diagnosed with grade I varices [33]. Variceal surveillance was largely suspended following the pandemic [34], but uTNE may have a substantial role to play during service recovery and in selected patients for whom pharmacological therapy with non-selective beta-blockers is contraindicated.
uTNE had previously been demonstrated in two small studies to have equivalent efficacy to cEGD in the detection and grading of esophageal varices [7]; this has since also been evidenced in a recent systematic review [35] which included seven studies, comprising 752 patients in total. It has also been shown to be efficacious in a further recent study by De Faria et al. [36], although direct comparison with cEGD was not made.

The use of sedation in cirrhotic patients was not considered in the previous reviews. Sedation has been acknowledged as potentially precipitating complications in patients with cirrhosis, such as hepatic encephalopathy [37, 38], therefore methods of variceal screening and surveillance which negate this requirement (such as uTNE) are preferable.

Previously identified concerns regarding coagulopathy [7] have hindered the adoption of uTNE in patients with cirrhosis, and in two studies patients who had platelets below $20 \times 10^{9} / \mathrm{L}$ [39] and $30 \times 10^{9} / \mathrm{L}$ were excluded [40]. Little new evidence has emerged in recent years regarding coagulopathy and uTNE, however, it is important to highlight that, in 2011 Choe et al. [39] noted the diagnostic accuracy of uTNE even in patients with marked diathesis (defined as platelets $<50 \times 10^{9} / \mathrm{L}$ and/or prothrombin time $\geq 1.7$ INR) and stated that appropriate pre-medication and use of a small diameter EGD was adequate in offsetting risk.

The inability to carry out variceal band ligation may be viewed as an outstanding limitation in the adoption of uTNE; however with recent evidence suggesting that primary prophylaxis with carvedilol is of equal efficacy to band ligation [41], the cohort of patients to which this may apply is limited. This, in addition to the lack of a requirement for sedation (with the resultant increased safety profile and reduced costs), equivalent diagnostic efficacy to cEGD and the widely reported increased tolerability (which may facilitate increased attendance among this high-risk population) suggest that uTNE should be considered in the majority of patients with cirrhosis.

\section{Eosinophilic Esophagitis (EoE)}

The use of uTNE in the diagnosis of EoE was not considered by previous reviewers. It has the potential to be used both diagnostically and in the follow-up of patients refractory to medical treatments. At present, there are three small studies that assess the use of uTNE in EoE; different endoscopes were used in each which makes drawing definitive conclusions regarding diagnostic accuracy difficult. Friedlander et al. [42] considered transnasal esophagoscopy in the monitoring of 21 pediatric patients. It was reported to be well-tolerated and the $2 \mathrm{~mm}$ and $1.2 \mathrm{~mm}$ biopsy forceps used were of equal efficacy to cEGD in obtaining the adequate epithelial surface area required for eosinophil count 


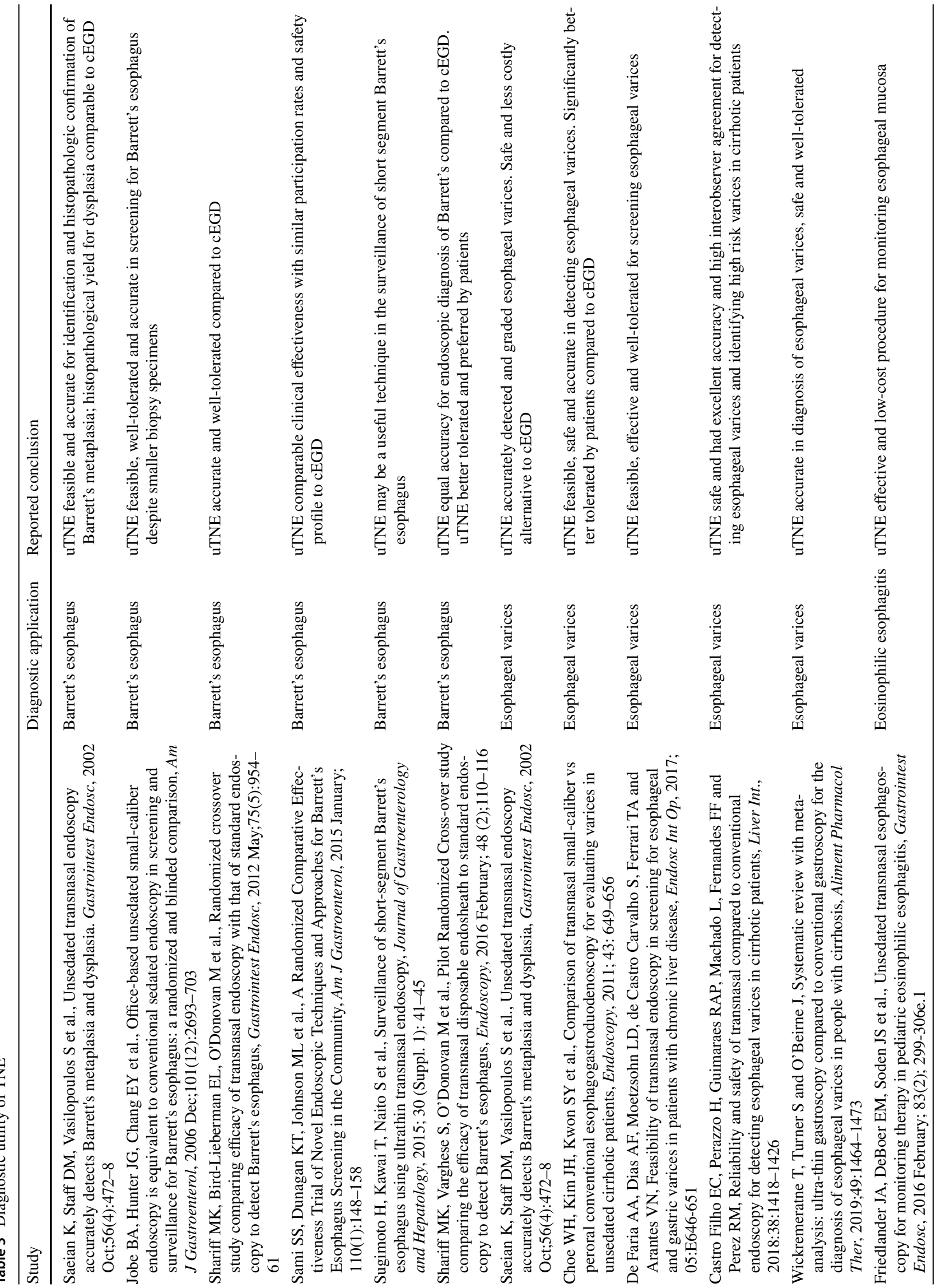


evaluation. Similarly, Philpott et al. [43], who considered transnasal gastroscopy in 24 adult patients, also reported good levels of tolerability and adequate histological sampling using $2 \mathrm{~mm}$ biopsy forceps. Both studies acknowledge the limitations of the biopsy forceps in obtaining adequate sampling of the lamina propria to allow grading of fibrosis; however, at present, this has not been standardized to form part of the routine clinical assessment. The theoretical concern of reduced diagnostic yield of EoE due to smaller biopsies can be addressed by taking extra biopsies. Further studies are needed in that respect.

It is notable that the only evidence for use of uTNE in pediatric patients is in those with EoE. A novel study by Nguyen et al. [44] considered the use of virtual reality video goggles as a distraction device during uTNE in 190 children. As in the aforementioned studies, visual and histological findings were adequate for assessment of EoE. In this study, and in a more recent review [45], particular attention is also drawn to the advantages of uTNE in the pediatric population, especially the reduced exposure to repeated general anesthetics which have previously been proposed as exposing children to risk of cardiopulmonary complications when undergoing cEGD. Parental anxiety regarding the procedure may consequently be reduced, as is time lost from education [46].

A further general advantage of uTNE is that it can transverse most tight strictures [16] and with minimal trauma, thus characterizing EoE-related strictures better. The combination of uTNE and bougie dilatation over a guidewire placed by uTNE has the potential to obviate the need for dilatations under fluoroscopic screening with obvious logistic advantages and less radiation; data are still required regarding this.

\section{Gastroduodenal}

\section{Gastric Cancer}

Many papers $[47,48]$ have discussed the possible reduction in the detection of early gastric cancers by UTNE compared to cEGD, and it was previously suggested that lesions in the upper stomach were more likely to be missed by uTNE [7]. Possible reasons for reduced detection included lower resolution, reduced field of view and lower light intensity of UTNE.

Due to their widespread screening program for gastric cancer, the majority of studies in this area have been carried out in Japan. Studies that have previously questioned the diagnostic utility of uTNE for early gastric cancers, include those of Hayashi et al. [47] and Toyoizumi et al. [48]; both studies used endoscopes without color enhancement (NBI, BLI or LCI), although they did acknowledge that application of such technologies to uTNE would likely reduce this 
apparent discrepancy in diagnosis of early gastric cancer lesions.

More recent studies have shown no significant differences in detection rates, which is likely a reflection of the number of technological advances in uTNE. In a retrospective study comparing early gastric cancer diagnosis by uTNE (Fuji) versus cEGD, Suzuki et al. [49] found similar detection rates. This study had included 129 'post-endoscopic submucosal dissection (ESD)' patients in both uTNE and cEGD groups, with a cancer detection rate of $7.8 \%$ and $7.0 \%$, respectively.

Yokoyama et al. [50], in a study involving 36 patients with known early gastric cancers, showed that UTNE with Flexible Spectral Imaging Color Enhancement (FICE) significantly improved the early gastric cancer detection rate to levels comparable to cEGD with white light endoscopy; they suggest that FICE compensated for any disadvantages of uTNE. These studies, however, are small and, although definitive conclusions may not be possible until further large studies become available, it is increasingly clear that the latest TNE endoscopes have enhanced diagnostic capabilities similar to most standard endoscopes. It should be noted that any discrepancy in the detection of early gastric cancer is heightened in patients at high risk of gastric cancer (e.g., ethnic predisposition, previous ESD of gastric cancer). uTNE is currently used in Japan to follow-up previous ESD for cancer, which suggests endoscopists there have confidence in its detection rate; this is likely influenced by the encouraging results reported with the use of BLI and LCI in cEGD [51] which can be extrapolated to uTNE, given that the same technologies are available. In a recent editorial (2021) [52], Kawai states that, due to good tolerability and fewer effects on cardiovascular function, it is expected that TNE endoscopes may actually be the first option for endoscopic screening of gastric cancer in older people. In the UK and the Western world few EGDs are carried out for gastric cancer screening and, although not much data on diagnostic yield is available, it is reasonable to assume that uTNE with advanced image enhancement is equivalent to cEGD [52].

\section{Celiac Disease}

Duodenal biopsies demonstrating villous atrophy remain essential for a diagnosis of adult coeliac disease [53], particularly in cases that anti-tissue transglutaminase antibodies are not sufficiently high to be at diagnostic levels. Duodenal biopsies obtained by TNE have been shown to be adequate for the purposes of histological diagnoses [54], with villous atrophy readily identifiable on samples obtained [13]. There are currently, however, no studies which consider the use of TNE specifically in the diagnosis or follow-up of celiac disease.

\section{uTNE in the Covid-19 Era}

The COVID-19 pandemic has had a significant impact on the delivery of upper GI endoscopy and has strengthened the argument for the greater use of uTNE. Despite the widespread use of vaccination, the emergence of COVID-19 viral mutations is likely to prolong the pandemic and delay the full recovery of endoscopic services, therefore the need for efficient endoscopic procedures allowing rapid turnover of patients is high in order to reduce waiting times.

uTNE has been shown to allow quicker throughput of patients without the need for the recovery time associated with sedation. As such it is likely to be a useful tool in helping more procedures to be done per unit time. Furthermore, uTNE has better cardiorespiratory tolerability and fewer cardiovascular adverse effects due to minimal increases in systolic blood pressure and less sympathetic stimulation when compared to cEGD [4]; it is also associated with negligible reductions in oxygen saturation [8], indicating that efficiency does not come at the cost of safety. Given the high prevalence of cardiovascular disease among Western populations [55], and the emergence of a proportion of COVID19 patients with compromised cardio-respiratory reserves, uTNE could perhaps be considered as the first line diagnostic option for such patients requiring an EGD. A recent German study [56] considered the use of uTNE during the COVID-19 pandemic; it demonstrated high levels of tolerability, satisfaction, shorter recovery times and reduced staff exposure when compared to cEGD.

As it seems likely that the COVID-19 virus will continue to circulate, there is a strong need to prepare now to minimize the risk of any further waves impacting on an already stretched service. Aerosol generation during endoscopy, putting endoscopy staff at risk [57], has been an important factor in limiting endoscopy provision. A study by Phillips et al. [58] (2021), that compared aerosol generation assessed via particle counters in 36 cEGDs and 11 uTNE procedures reported that aerosol generation in UTNE was approximately half that found in cEGD procedures; this is thought be due to less gagging [4]. Aerosol generation could be reduced further by using a surgical mask over the mouth during uTNE. A short paper by Higashimori et al. [59] (2021) using a surgical mask over the mouth while performing uTNE showed that this significantly reduced the spread of fluorescent dye droplets from a mannequin cough simulation device. Without a mask, the dye spread up to $1.5 \mathrm{~m}$ from the mannequin, with a mask the dye was limited to the inside of the mask only. They have subsequently used this approach without any safety issues in 732 uTNE patients. Lazaridis et al. [60] also report use of a 'double-surgical-mask-with-slit' method to reduce exposure to aerosol generation at upper GI endoscopy; this has been adopted by many UK centers 
for transnasal endoscopy. Additionally, overall risk to staff would be reduced with uTNE if only one assistant entered the room rather than the standard of two that is needed for cEGD.

It is, of course, self-evident that use of uTNE does not negate the need for pre-procedure patient testing and appropriate personal protective equipment (PPE), as recommended by national guidelines.

\section{Cost-Effectiveness}

uTNE has previously been shown to be more cost-effective than cEGD [7, 61]; this was primarily a result of direct savings due to the lack of requirement for sedation, oxygen (and subsequent monitoring), in addition to reduced recovery time needed. Indirect savings were also noted as playing an important role [12, 62], with patients able to return to their place of employment on the same day as the procedure and a further individual not being required to take time off work to accompany a previously sedated patient home.

More recent literature [12] has demonstrated that the reduced costs associated with uTNE increase the possibility for its adoption in screening programs (e.g., for Barrett's esophagus) in community settings; Moriarty et al. [63] highlighted the savings (both direct and indirect) associated with community mobile van unit uTNE when compared to hospital-based uTNE and cEGD.

Evidence concerning cost-effectiveness of uTNE continues to be encouraging and is particularly relevant in the COVID-19 era, however, there remains a need for a robust cost-benefit analysis comparing it to cEGD. At present, there are no UK data readily available to allow for a direct comparison in cost to be made, although a recently published report from the United States is suggestive of significant potential savings [64]. Such data will be important in increasing the uptake of uTNE.

\section{Current Status of UTNE in the UK}

The national census of UK endoscopy services in 2019 [3] reported the number of UTNE procedures carried out as 26,685 ; this compares to 866,844 cEGDs (the number of which were purely diagnostic was not recorded). These data, obtained from the National Endoscopy Database, are due to be updated in the next twelve months and, it is envisaged, would capture the impact that the pandemic has had. Data available from two endoscope manufacturers (Fujifilm and Olympus) suggest an upward trend in uTNE sales over the past year (2020-2021) and it will be important to ascertain if this increase is also replicated in the number of procedures performed. Furthermore, regional case studies $[65,66]$ have shown that UTNE can easily be adopted by UK clinicians experienced in cEGD and that it is well tolerated by patients. In the absence of formal guidelines, such studies may prove to be influential in providing a blueprint for other UK centers considering uTNE.

\section{Conclusion}

While the Western world and, more specifically, the UK, has been slower to adopt uTNE than countries such as Japan, the COVID-19 pandemic has acted as an impetus to potentially increase its use [56]. Advances in imaging technology (e.g., BLI and LCI), coupled with an increasing body of evidence [47-50] suggesting that diagnostic accuracy in gastric cancer may be equivalent to cEGD, go some way to address concerns [7] that have previously impeded its use. In esophageal conditions uTNE has the potential to be a welltolerated screening tool among high-risk populations; with increasing availability of disposable devices there is also the possibility of opening up this technology to primary care and community settings.

The lack of a formal training program in uTNE may continue to act as a limiting factor in its use in more centers. In the UK the Joint Advisory Group (JAG) does however, advise endoscopists to attend a number of training courses that are available [67]. The development of formal guidelines, regarding both training and the use of uTNE in a purely diagnostic setting, would play a significant role in increasing its use; this would enable more endoscopists to confidently incorporate it within their centers, thus offering a larger proportion of patients a less distressing and more convenient alternative to cEGD.

Acknowledgments Many thanks to Mr Stephen Arundel and Mr Matthew Aronow, Healthcare 21 Group UK, Mr Nick Schneeberger and Mr Daniel Wallaker, Olympus Medical UK and Mr Idris Bora, Pentax Medical UK. Thanks also to Mr Stephen Liddell, Creative82, and Dr S Inglis, Department of Medical Physics, Royal Infirmary of Edinburgh, for the illustrations, and to Mrs Jennifer Berks for her kind assistance.

Author's contribution RKG and WMB both contributed to the literature review, design, writing and revision of the article. ARR assisted in the writing and revision of the article. RK critically revised the article. JNP was senior author and critically revised the article for important intellectual content. All authors approved the final version of the manuscript.

\section{Declarations}

Conflict of interest JNP has received research and educational support from Aquilant Limited. RKG, WMB, ARR and RK have no conflicts of interest to declare. 


\section{References}

1. Shaker R. Unsedatated trans-nasal pharyngoesophagogastroduodenoscopy (T-EGD): technique. Gastrointest Endosc 1994;40:346-348.

2. Tanuma T, Morita Y, Doyama H. Current status of transnasal endoscopy worldwide using ultrathin videoscope for upper gastrointestinal tract. Digestive Endoscopy 2016;28:25-31.

3. Ravindran S, Bassett P, Shaw T et al. National census of UK endoscopy services in 2019. Front Gastroenterol 2020;3:1-10.

4. Alexandridis E, Inglis S, McAvoy NC et al. Randomised clinical study: comparison of acceptability, patient tolerance, cardiac stress and endoscopic views in transnasal and transoral endoscopy under local anaesthetic. Aliment Pharmacol Ther 2020;40:467-476.

5. Despott EJ, Baulf M, Bromley J, Gupta S, Fraser C. Scent: final report of the first UK prospective, randomised, head-to-head trial of transnasal vs oral upper gastrointestinal endoscopy. Gut 2010;59:A24.

6. Sami SS, Subramanian V, Ortiz-Fernández-Sordo J et al. Performance characteristics of unsedated ultrathin video endoscopy in the assessment of the upper GI tract: systematic review and meta-analysis. Gastrointest Endosc 2015;82:1005.

7. Parker C, Alexandridis E, Plevris J, O'Hara Panter S. Transnasal endoscopy: no gagging no panic! Front Gastroenterol 2016;7:100.

8. Atar M, Kadayifci A. Transnasal endoscopy: technical considerations, advantages and limitations. World J Gastrointest Endosc 2014;6:41-48.

9. Iwamoto J, Mizokami Y, Shimokobe K et al. Pretreatment methods in transnasal endoscopy. Hepatogastroenterology 2011;58:842-845.

10. Mieda M, Miyashita $\mathrm{H}$, Osawa $\mathrm{H}$ et al. Risk factors for nasal bleeding in patients undergoing transnasal gastrointestinal endoscopy. Kaohsiung J Med Sci 2018;34:295-300.

11. Hu C-T, Lei W-Y, Lin J-S, Hung J-S. Endoscopic meatus scoring scale versus sniff test to predict insertability before transnasal endoscopy: a prospective randomized study. J Gastroenterol Hepatol 2017;32:1914-1921.

12. Blevins $\mathrm{CH}$, Iyer PG. Putting it through the nose: the ins and outs of transnasal endoscopy. Am J Gastroenterol 2016;111:1371-1373.

13. Walter T, Chesnay A-L, Dumortier J et al. Biopsy specimens obtained with small-caliber endoscopes have comparable diagnostic performances than those obtained with conventional endoscopes. J Clin Gastroenterol 2010;44:198.

14. Kawai T, Yanagizawa K, Naito $S$ et al. Evaluation of gastric cancer diagnosis using new ultrathin transnasal endoscopy with narrow-band imaging: preliminary study. J Gastroenterol Hepatol 2014;29:1007.

15. Marlicz W, Ren X, Robertson A et al. Frontiers of robotic gastroscopy: a comprehensive review of robotic gastroscopes and technologies. Cancers 2020;12:10076.

16. Aydinli M, Koruk I, Dag MS, Savas MC, Kadayifci A. Ultrathin endoscopy for gastrointestinal strictures. Dig Endosc 2012;24:150-153.

17. Kamani L, Makhejani KR, Ahmad BS. Safety of trans-nasal percutaneous endoscopic gastrostomy placement. J College Phys Surg Pak 2017;27:751-753.

18. McCulloch A, Roy O, Massey D et al. Nasal unsedated seated percutaneous endoscopic gastrostomy (nuPEG): a safe and effective technique for percutaneous endoscopic gastrostomy placement in high-risk candidates. Front Gastroenterol 2018;9:105-109.

19. Yuki M, Amano Y, Komazawa Y, Fukyhara H et al. Unsedated transnasal small-caliber esophagogastroduodenoscopy in elderly and bedridden patients. World J Gastroenterol 2009;15:5586-5591.

20. Külling D, Bauerfeind P, Fried M. Transnasal versus transoral endoscopy for the placement of nasoenteral feeding tubes in critically ill patients. Gastrointest Endosc 2000;52:506-510.

21. Kikuchi D, Tanaka M, Nakamura $S$ et al. Feasibility of ultrathin endoscope for esophageal endoscopic submucosal dissection. Endosc Int Open 2021;09:E606-E609.

22. Masakatsu N, Shiroeda H, Tahara T, Shibata T, Arisawa T. Endoscopic submucosal dissection of an esophageal tumour using a transnasal endoscope. Endoscopy 2014;46:E115-E116.

23. Lee S-Y, Kawai T. Transnasal route: new approach to endoscopy. Gut Liver 2008;2:155-165.

24. Xavier AT, Alvares AV, Iyer PG, Arantes VN. Unsedated transnasal endoscopy for preoperative examination of bariatric patients: a prospective study. Obes Surg 2020;30:238-243.

25. DeBoer AM, Mellion KM, Frankki SM et al. Pre-screening for bariatric surgery patients: comparative effectiveness of transnasal endoscopy versus esophagogastroduodenoscopy. Surg Endosc 2021;35:4153-4159.

26. Abe H, Kamimura K, Arao Y, Kohisa J, Terai S. Advances in the treatment of gastrointestinal bleeding: safety and efficiency of transnasal endoscopy. Medicines 2021;8:53.

27. Fitzgerald RC, di Ragunath PM et al. British Society of Gastroenterology guidelines on the diagnosis and management of Barrett's oesophagus. Gut 2013;7:1-36.

28. Sami SS, Dunagan KT, Johnson ML et al. A randomized comparative effectiveness trial of novel endoscopic techniques and approaches for Barrett's esophagus screening in the community. Am J Gastroenterol 2015;110:148-158.

29. Shariff MK, Varghese S, O'Donovan $M$ et al. Pilot randomized cross-over study comparing the efficacy of transnasal disposable endosheath to standard endoscopy to detect Barrett's oesophagus. Endoscopy 2016;48:110-116.

30. Sugimoto H, Kawai T, Naito $\mathrm{S}$ et al. Surveillance of short-segment Barrett's esophagus using ultrathin transnasal endoscopy. J Gastroenterol Hepatol 2015;30:41-45.

31. Takeda $T$, Nagahara $A$, Ishizuka $K$ et al. Improved visibility of Barrett's esophagus with linked color imaging: inter-and intra-rater reliability and quantitative analysis. Digestion 2018;97:183-194.

32. Fitzgerald RC, di Pietro M, O’Donovan M et al. Cytospongetrefoil 3 versus usual care to identify Barrett's oesophagus in a primary care setting: a multicentre, pragmatic, randomised controlled trial. Lancet 2020;396:333-344.

33. Tripathi D, Stanley AJ, Hayes PC et al. UK guidelines on the management of variceal haemorrhage in cirrhotic patients. Gut 2015;64:1680-1704.

34. British Society of Gastroenterology, Endoscopy Activity and COVID-19. https://www.bsg.org.uk/covid-19-advice/endos copy-activity-and-covid-19-bsg-and-jag-guidance/. Accessed 09 Aug 2021

35. Wickremeratne T, Turner S, O'Beirne J. Systematic review with meta-analysis: ultra-thin gastroscopy compared to conventional gastroscopy for the diagnosis of oesophageal varices in people with cirrhosis. Aliment Pharmacol Ther 2019;49:1464-1473.

36. De Faria AA, Dias AF, Moetzsohn LD, de Castro Carvalho S, Ferrari TA, Arantes VN. Feasibility of transnasal endoscopy in screening for esophageal and gastric varices in patients with chronic liver disease. Endosc Int Op 2017;05:E646-651.

37. Haq MM, Faisal N, Khalil A, Haqqi SAH, Shaikh H, Arain N. Midazolam for sedation during diagnostic or therapeutic upper gastrointestinal endoscopy in cirrhotic patients. Eur J Gastroenterol Hepatol 2012;24:1214-1218. 
38. Triantafillidis JK, Merikas E, Nikolakis D, Papalois AE. Sedation in gastrointestinal endoscopy: current issues. World J Gastroenterol 2013;19:463-481.

39. Choe WH, Kim JH, Kwon SY et al. Comparison of transnasal small-caliber vs peroral conventional esophagogastroduodenoscopy for evaluating varices in unsedated cirrhotic patients. Endoscopy 2011;43:649-656.

40. Castro Filho EC, Perazzo H, Guimaraes RAP, Machado L, Fernandes FF, Perez RM. Reliability and safety of transnasal compared to conventional endoscopy for detecting oesophageal varices in cirrhotic patients. Liver Int 2018;38:1418-1426.

41. Dwinta M, Putera DD, Adda'i MF, Hidayat PN, Hasan I. Carvedilol vs endoscopic variceal ligation for primary and secondary prevention of variceal bleeding: systematic review and metaanalysis. World J Hepatol 2019;11:464-476.

42. Friedlander JA, DeBoer EM, Soden JS et al. Unsedated transnasal esophagoscopy for monitoring therapy in pediatric eosinophilic esophagitis. Gastrointest Endosc 2016;83:299-306e.1.

43. Philpott H, Nandurkar S, Royce SG, Gibson PR. Ultrathin unsedated transnasal gastroscopy in monitoring eosinophilic esophagitis. J Gastroenterol Hepatol 2016;31:590-594.

44. Nguyen N, Lavery WJ, Capocelli KE et al. Transnasal endoscopy in unsedated children with Eosinophilic esophagitis using virtual reality video goggles. Clin Gastroenterol Hepatol 2019;17:2455-2462.

45. Nguyen N, Mark J, Furuta GT. Emerging role of transnasal endoscopy in children and adults. Clin Gastroenterol and Hepatol 2021;17:S1542-3565(21)01259-3.

46. Thakkar K, El-Serag HB, Mattek N, Gilger MA. Complications of pediatric EGD: a 4-year experience in PEDS-CORI. Gastrointest Endosc 2007;65:213-221.

47. Hayashi Y, Yammamoto ST et al. Comparison of the diagnostic utility of the ultrathin endoscope and the conventional endoscope in early gastric cancer screening. Dig Endosc 2009;21:116-121.

48. Toyoizumi $\mathrm{H}$, Kaise $\mathrm{M}$, Arakawa $\mathrm{H}$ et al. Ultrathin endoscopy versus high-resolution endoscopy for diagnosing superficial gastric neoplasia. Gastrointest Endosc 2009;70:240-245.

49. Suzuki T, Kitagawa Y, Nankinzan R, Yamaguchi T. Early gastric cancer diagnostic ability of ultrathin endoscope loaded with laser light source. World J Gastroenterol 2019;25:1378-1386.

50. Yokoyama T, Miyahara R, Funasaka $\mathrm{K}$ et al. The utility of ultrathin endoscopy with flexible spectral imaging color enhancement for early gastric cancer. Nagoya J Med Sci 2019;81:241-248.

51. Shinozaki S, Osawa H, Hayashi Y, Lefor AK, Yamamoto H. Linked color imaging for the detection of early gastrointestinal neoplasms. Therap Adv Gastroenterol 2019;12:1756284819885246.

52. Kawai T. Improved transnasal examination of the upper gastrointestinal tract through advancements in ultrathin endoscopes. Dig Endosc 2021;3:10007.

53. Ludvigsson JF, Bai JC, Biagi F et al. Diagnosis and management of adult coeliac disease: guidelines from the British Society of Gastroenterology. Gut 2014;63:1210-1228.

54. Al-Karawi MA, Sanai FM, Al-Madani A, Kfoury H, Yasawy MI, Sandokji A. Comparison of peroral versus ultrathin transnasal endoscopy in the diagnosis of upper gastrointestinal pathology. Ann Saudi Med 2000;20:3-4.
55. British Heart Foundation, Facts and Figures, https://www.bhf.org. uk/what-we-do/news-from-the-bhf/contact-the-press-office/factsand-figures. Accessed 16 Sep 21

56. Neumann H, Vieth M, Neumann SH. Transnasal endoscopy during Covid-19 pandemic: a safe and feasible alternative. United Eur Gastroenterol J 2020;8:74.

57. British Society of Gastroenterology rationale around current advice to all Endoscopy Units, first published 25 Mar 2020, https://www.bsg.org.uk/covid-19-advice/bsg-rationale-aroundcurrent-advice-to-all-endoscopy-units/. Accessed 19 June 21

58. Phillips F, Crowley J, Warburton S, Gordon GSD, Parra-Blanco A (2021) Quantifying aerosol and droplet generation during upper and lower gastrointestinal endoscopy: whole procedure and eventbased analysis, https://www.medrxiv.org/content/https://doi.org/ 10.1101/2021.04.15.21255544v1. Accessed 19 June 21 [Preprint]

59. Higashimori A, Maruyama H, Nadatani Y, Fujiwara Y. The "new normal" following the Covid-19 pandemic: a simple infectionprevention measure using a surgical mask during transnasal endoscopy. Clin Endosc 2021;3:1-3.

60. Lazaridis N, Skamnelos A, Murino A, Chacchi CR, Koukias N, Despott EJ. Double-surgical-mask-with-slit" method: reducing exposure to aerosol generation at upper gastrointestinal endoscopy during the COVID-19 pandemic. Endoscopy 2020;52:982-929.

61. Bampton PA, Reid DP, Johnson RD, Fitch RJ, Dent J. A comparison of transnasal and transoral oesophagogastroduodenoscopy. $J$ Gastroenterol Hepatol 1998;13:579-584.

62. Hu C-T. Let your patients watch and talk during examination: a review of unsedated transnasal endoscopy. Tzu Chi Med J 2013;7:139-145.

63. Moriarty JP, Shah ND, Rubenstein JH et al. Costs associated with Barrett's esophagus screening in the community: an economic analysis of a prospective randomized controlled trial of sedated versus hospital unsedated versus mobile community unsedated endoscopy. Gastrointest Endosc 2018;87:88-94.e2.

64. Sami S, Moriaty JP, Rosedahl JK et al. Comparative cost effectiveness of reflux-based and reflux-independent strategies for barrett's esophagus screening. Am J Gastroenterol 2021;116:1620-1631.

65. McColl G, Yeap E, Stirling L, Sharp C, Robertson K. Introduction of transnasal endoscopy to a scottish district general hospital. Gastroint Nurs 2021;44:71.

66. James Cook University Hospital launches new Transnasal Endoscopy Service, https://www.ahsn-nenc.org.uk/wp-content/uploads/ 2019/10/Olympus-JCUH-Case-Study-Final-v4.pdf. Accessed 26 July 21

67. The JAG accreditation scheme, JAG guidance: transnasal endoscopy, October 2018, https://www.thejag.org.uk/CMS/UploadedDo cuments/Scheme/Scheme5/Guidance/191011\%20-\%20guidance\% 20-\%20transnasal\%20endoscopy.pdf. Accessed 03 July 21

Publisher's Note Springer Nature remains neutral with regard to jurisdictional claims in published maps and institutional affiliations. 